\title{
Changes in Liver Cell Apoptosis after Vaccination by Schistosomulae Antigen and/or Artesunat Treatment in Murine Schistosoma Mansoni
}

\author{
Magda A. Elsettawy and Shereen M. Ibrahim \\ Department of Medical Parasitology, Faculty of Medicine, Zagazig University,Egypt
}

\section{Corresponding Author} Mageda A. Elsettawy

Mobile:

$+201006224326$

E mail: magdasat9@gmail. com

Key words: Schistosoma mansoni, SLAP, ART, IL12, Parasitological parameters, liver cell apoptosis
Background and study aim: The target for a schistosoma vaccine or treatment is the reduction in morbidity. The hope for vaccine or treatment is to decrease worm burden and egg pathology to the least percentage, this work aimed to compare between schistosomulae lung stage antigen (SLAP) with and without adjuvant (IL12) and artesunate (ART) treatment regarding their effect especially on liver cell apoptosis.

Materials and Methods: Schistosomulae were extracted from the lungs 18 days post infection with Schistosoma mansoni. Eight groups of Swiss albino mice (8 mice each group) were classified as follows: Group A was served as healthy control group, group B was infected with \pm 100 cercariae and served as infected control group, group $\mathrm{C}$ infected then treated by artesunate monotherapy $(400 \mathrm{mg} / \mathrm{kg})$ single oral dose 6 weeks post infection., group D was immunized with 50ug of SLAP then infected, group E infected then treated by artesunate monotherapy (400 mg/kg) single oral dose 3 weeks post infection, group F was immunized with 50ug of SLAP and 100ug of IL12,group G was immunized with 50ug of SLAP and 100ug of IL12 then infected then treated by artesunate monotherapy ( $400 \mathrm{mg} / \mathrm{kg}$ ) single oral dose 6 weeks post infection, group $\mathrm{H}$ was immunized with 50ug of SLAP and 100ug of IL12 infected then treated by artesunate monotherapy (400 mg/kg) single oral dose 3 weeks post infection. All groups vaccinated with SLAP vaccine were given booster doses after2 weeks of the first doses. Seven weeks post infection mice were sacrificed for parasitological parameters measurements (worm burden, tissue egg load and oogram pattern), histopathological and immunohistochemical studies using P53 and Bcl-2 markers for determination of liver cells apoptosis.

Results and Conclusion: Apoptosis detected by immunohistochemical studies is a good evaluation method. IL12 potentiated the protective effect of SLAP vaccine, ART 3 weeks post infection is better than 6 week post infection in reduction of liver pathology and apoptosis. The combination of both (SLAP+IL12+ART 3 weeks post infection) is the best regarding the reduction of tissue egg count, worm burden, hepatic immunopathology and apoptosis.

\section{INTRODUCTION}

Schistosomiasis represents the third most tropical disease in the world, especially in developing countries in Africa, South America, the Caribbean, the Middle East and Asia [1]. Praziquantel (PZQ) provides the main drug for schistosomiasis morbidity control. Its low cost, safety and efficacy against adult worms of all schistosome species led to its widespread use [2]. Appearance of PZQ-resistant strains may put the drug out of use [3]. Moreover, PZQ fails to prevent re- infection and has minor activity against immature worms [4], which can result in low cure rates in areas where schistosomiasis is hyper endemic [5]. Inyang-Etohet al. [6] approved that artesunate (ART), one of the artemisinin group is a promising drug for treatment and chemoprophylaxis of schistosomiasis, they are specifically effective against young developing forms of schistosomes [7].

vaccine strategies represent an essential component for the future control of schistosomiasis as a complement to 
chemotherapy. [8]. Control based on chemotherapy followed by vaccination would result in a short term effect with long-term protection [9]. The efficacy of schistosoma vaccine relies on the stage of the parasite and the adjuvant used [10]

Antigens obtained from lung-stage shistosomulae are more efficient stimulators of Th1 cytokines than those from cercariae and skin-stage larvae [11]. Th1 cytokines is considered the protective immunity [12].

Several adjuvants including CFA, BCG and ISA $70 \mathrm{M}$ used as a complementary to shistosome vaccin [13]. Moreover IL12 potentiates the protective effect of a vaccine [14]. The use of IL-12 in addition to $S$. mansoni soluble worm antigen or egg antigen was found to reduce granuloma formation [15] and modulation of Th1/Th2 responses [16]. IL-12 was also reported to reduce hepatic granuloma size, enhance protection by $\mathrm{Sm} 14$ (rSm14) vaccination in mice [17]. IL-12 is beneficial for the promotion of humoral and cell-mediated protection against schistosomiasis, especially when used as a complementary to plasmid DNA produced a significant immune response to $S$. japonicum [18]. Also immunization with $\mathrm{pcDNA} / \mathrm{SjGST}$ vaccine, pIL-12, and rSjGST has significant protective effect in mice [19].

Apoptosis is a programmed cell death which has a major role in balancing cell proliferation and remodeling tissue activities in many organisms [20]. Schistosoma mansoni eggs can suppress the activation of hepatic cells and soluble egg antigens also can promote immunocyte and liver cells apoptosis, as well as induction of morphological changes in the hepatic cells, inhibition of cell proliferation and arresting cell-cycle at the $G_{1}$ phase [21]. P53 tumor suppressor protein acts as a protector of genomic activity by induction of either cell cycle arrest (at G1 and/or G2 phase) or programmed cell death (apoptosis) [22]. P53 is implicated in the induction of both apoptotic signal pathways leading to activation caspases that mediate apoptosis, the extrinsic pathway involves engagement of certain death receptors that belong to the tumor necrosis factor receptor and, through the formation of death-inducing-signaling-complex [23]. This leads to a cascade of activation of caspases, including caspase-3 and caspase-8, which induce apoptosis. The intrinsic pathway is produced in response to DNA damage and is associated with mitochondrial depolarization and release of cytochrome $\mathrm{c}$ from the mitochondrial inter membrane space into the cytoplasm. Cytochrome c cause activation of caspase- 3 , caspase- 6 and caspase-7 [24]. Proteins of the Bcl-2 are considered the key regulators of the intrinsic pathway [25]. Bcl-2 comprise subgroups of pro survival proteins that promote either cell survival or death, These mediators of cell death initiate the apoptotic cascade by forming pore like structures in the outer mitochondrial membrane, leading to release of apoptogenic factors, which enables the activation of caspases and other proteolytic enzymes responsible for destructing the cell [26]. A high level of Bcl-2 expression prevents cells from apoptosis, so it is protective in high level [27]. $\mathrm{Bcl}-2$ gene is an anti apoptotic protein, participate in the p53 apoptotic pathway and the equilibrium between those positively and negatively regulatory proteins is essential for the susceptibility to apoptosis [28]. In an attempt to introduce an effective anti schistosomal vaccine and/or treatment, the present study aimed to evaluate the effect of artesunat treatment and/or schistosomal lung antigen preparations (SLAP) with and without IL-12 or combination of them ,to assess also their protective and curative role reflected by liver cell apoptotic changes.

\section{MATERIALS AND METHODS}

\section{Type of study:}

This non randomized, control-trial study was performed at the laboratories of the Parasitology Department, Faculty of Medicine, Zagazig University and Theodor Bilharz Research Institute (TBRI), Giza, Egypt, from December2016 to December 2017.

\section{Experimental design:}

Eight groups of Swiss albino mice (8 mice, each group) were classified as follows: Group A was non immunized and served as healthy control group, group B was infected with 100 cercaria and served as infected control group, group C infected then treated by artesunate monotherapy $(400 \mathrm{mg} / \mathrm{kg}$ ) single oral dose 6 weeks post infection, group D was immunized with 50ug of SLAP then infected, group $\mathrm{E}$ infected then treated by artesunate monotherapy $(400 \mathrm{mg} / \mathrm{kg})$ single oral dose 3 weeks post infection, group F was immunized with 50ug of SLAP and 100ug of IL12, group $G$ was immunized with 50ug of SLAP and 100ug of IL12 then infected then treated by artesunate monotherapy $(400 \mathrm{mg} / \mathrm{kg})$ single oral dose 6 weeks post infection, group $\mathrm{H}$ was immunized with 50ug of SLAP and 100ug 
of IL12 infected then treated by artesunate monotherapy $(400 \mathrm{mg} / \mathrm{kg}$ ) single oral dose 3 weeks post infection. All groups vaccinated with SLAP vaccine were given booster doses after 2 weeks of the first doses. Seven weeks post infection mice were sacrificed for worm burden, oogram pattern, removal of the livers and intestine was done, the intestine was used for the determination of tissue egg load, and the liver was subdivided into two pieces the first was a small piece used for the determination of tissue egg load and the second large piece of liver tissue was fixed in $10 \%$ formalin for histopathological and immunohistochemical studies.

\section{Preparation of schistosomula lung antigens and IL12 :}

Each B. alexandrina snail obtained from Theodor Bilharz, GIZA, Egypt, was placed singly with 8-10 miracidia to be infected, Collection of cercariae after nearly 28 days according to Liang et al. [29]. Seventy male Swiss albino mice were infected each with \pm 100 cercariae subcutaneously. The infective cercariae were suspended in $2 \mathrm{ml}$ distilled water [30]. Schistosomula were recovered from the lungs by a slight modification of the perfusion technique reported by Mangold et al. [31]. The previous isolated schistosomula were suspended in $15 \mathrm{ml}$ of Triton-EDTA buffer. The suspension was placed in ice and homogenized by sonication for 10 minutes, followed by centrifugation at 3000 (r.p.m) for 30 minutes, and so the antigenic preparation of schistosomulum according to Call et al. [32]. The protein content of SLAP was determined by Bradford method [33].

IL12 (catalog-KMC0122:2) were of high analytical grade, products of Sigma (Saint Louis, USA).

\section{Antigen administration:}

Each mouse was sensitized with a single subcutaneous injection of the selected antigen in a dose of $50 \mu$ suspended in $1 \mathrm{ml}$ of triton edta buffer. After 15 days, a second inoculation with the same antigen concentration was performed; thus, each mouse received a total dose of $100 \mathrm{~g}$ protein.

\section{Infection procedure:}

Two weeks after the last dose of vaccination, all mice were challenged with $100 \mathrm{~S}$. mansoni cercariae subcutaneously injected by an insulin syringe.

\section{Treatment protocol:}

Artesunate (ART) Sigma-Aldrich was suspended in a solution of $3 \%$ ethanol and $7 \%$ Tween 80 and water just before treatment [34]. It was administered orally to each mouse by gavage through mouth [35].

\section{Assessment by parasitological parameters:}

A- Worm burden: Worms recovered by liver and intestinal perfusion [36].

B- Tissue egg load: The number of eggs/gram tissues (liver and intestine) [37].

C- Oogram : The percentage of eggs at various developmental stages in the small intestine was determined [38].

\section{Assessment by histopathological studies:}

Parts of the livers of all studied groups were fixed in $10 \%$ formalin, paraffin section $4 \mu$ thickness cut and stained with Haematoxyline and Eosin to detect granuloma number and size, the number of hepatic granulomas was determined in five randomly selected low power microscopic fields. Three liver sections were examined for each mouse. The mean number of hepatic granulomas/liver section was calculated. The diameter of granulomas was measured using an ocular micrometer, this was done only for granulomas containing ova in the center, two perpendicular maximal diameters were measured to get the mean diameter for each granulomae, then the percentage reduction in the mean granuloma diameter (MGD) or number (no) were calculated according to the equation as follows: MGD or no of control infected group - MGD or no of treated group divided by MGD or no of control infected group [39].

Assessment by immunohistochemical studies: liver sections were examined using P53 and Bcl2 markers for determination of the extent of hepatocytes insult and detection of the degree of liver tissue apoptosis. These sections from all different studied groups were immunohistochemically stained for P53 and Bcl-2 using the Dako autostainer (Thermo fissure, USA). Then the slides were examined using a Zeiss light microscope (Oberkochen, Germany). P53 and Bcl2 expression sites were examined in hepatocytes, Kupffer cells and bile duct epithelium, their expression was assessed according to the percentage of positive cells, then the immunoreactive cells were semi-quantitatively estimated in five successive fields of maximum staining intensity of each section at 400x magnification and the mean values were calculated and the final value 
represented the mean of 80 readings per group [40]. Image analysis was adjusted using PAX-it image analysis software according to Tousson et al. [28]. In the present work, the use of P53,BcL2 histochemical technique as a marker of liver cell apoptosis . The criteria for a positive reaction confirming the presence of $\mathrm{p} 53$ and $\mathrm{Bcl}-2$ proteins is a dark, brownish, intra cytoplasmic precipitate. The changes in P53, Bcl-2 immuno reactivities expressions in different studied groups are graded according to the extension and density of the dark, brownish precipitate appeared at the positive slides into 4 grades as follows :

$0=$ negative to very mild reaction $(0-1 \%)$.

$1=$ mild reaction $(2-5 \%)$.

$2=$ moderate reaction $(6-10 \%)$.

$3=$ strong reaction $(>10 \%)$

\section{Ethical consideration:}

The mice used in this study were maintained according to the research protocols following the recommendations of the National Institutes of Health Guide for Care and Use of Laboratory Animals and as approved by ethics committee of the Faculty of Medicine, ZagazigUniversity.

\section{Acknowledgment :}

The authors are deeply indebted to Dr. Heba Khalil Mohamed, lecturer of Pathology Department, Theodor Bilharz Research Institute (TBRI), Imbaba, Giza for her unlimited help and moral support.

\section{RESULTS}

Evaluation of mean number of worm burden (Table 1) showed significant reduction $(P<0.05)$ between those treated by artesunat $3 \mathrm{w}$ post infection (group E) and infected non treated control, group B. The most significant reduction was in group $\mathrm{H}$ vaccinated by SLAP + IL12+ART 3 week post infection $(P<0.001)$. Statistically insignificant results $(P>0.05)$ were obtained between group C treated by ART 6 week post infection and group $D$ vaccinated with SLAP. While $P<0.05$ significance was between group $\mathrm{E}$ treated by ART 3 week post infection and group F vaccinated by SLAP+IL12.

Reduction of mean egg counts/gram liver (Table $2)$ showed significant reduction $(P<0.01)$ between those treated by artesunt $3 \mathrm{w}$ post infection (group E) and infected non treated control, group $\mathrm{B}$. The most significant reduction was in group $\mathrm{H}$ vaccinated by SLAP +IL12+ART 3 week post infection $(P<0.001)$. Statistically significant results $(P<0.05)$ were obtained between group $\mathrm{C}$ treated by ART 6 week post infection and group $D$ vaccinated with SLAP. While $P<0.01$ significance was between group E treated by ART 3 week post infection and group F vaccinated by SLAP+ IL12.

Regarding of mean egg counts/gram intestine (Table 3) showed significant reduction $(P<0.01)$ between those treated by artesunt $3 \mathrm{w}$ post infection (group E) and infected non treated control, group $\mathrm{B}$. The most significant reduction was in group $\mathrm{H}$ vaccinated by SLAP +IL12+ ART 3 week post infection $(P<0.001)$. Statistically significant results $(P<0.05)$ were obtained between group $C$ treated by ART 6 week post infection and group D vaccinated with SLAP. While $P<0.001$ significance was between group $E$ treated by ART 3 week post infection and group F vaccinated by SLAP+IL12.

Concering oogram, there was a decrease in the percentage of mature stages in the immunized and treated groups compared with the nonimmunized infected control group (Table 4). The decrease was significant in groups $\mathrm{C}$ and $\mathrm{D}$ $(\mathrm{P}<0.05)$, and in groups $\mathrm{E}$ and $\mathrm{F}(\mathrm{P}<0.01)$, and in group $\mathrm{G}(\mathrm{P}<0.001)$. There was a decrease in the percentage of immature stages in combined immunized groups with

regarding dead ova, there was an increase in the percentage of dead ova in all the immunized and treated groups compared with the nonimmunized infected control group. The increase was insignificant in groups $C$ and significant in group $\mathrm{D}(\mathrm{P}<0.05)$, in groups $\mathrm{F}, \mathrm{G}, \mathrm{H}(\mathrm{P}<0.001)$. The percentage of immature ova was decreased in all immunized groups compared with the nonimmunized infected control group.

As regard the mean granuloma number, the reduction was insignificant in group $\mathrm{C}(\mathrm{P}>0.05)$, significant in groups $\mathrm{D}(\mathrm{P}<0.05)$, and in Groups $\mathrm{F}, \mathrm{G}, \mathrm{H}(\mathrm{P}<0.001)$, with highest reductionin group $\mathrm{H}(90 \%)$ (Table 5 and Figs 2F) Whereas the reduction in granuloma size was insignificant in group $\mathrm{C}(\mathrm{P}>0.05)$; it was significant in groups $\mathrm{D}$ $(\mathrm{P}<0.05)$, in groups $\mathrm{F}, \mathrm{G}, \mathrm{H} \quad(\mathrm{P}<0.001)$ with highest reduction (97.8\%) in group $\mathrm{H}$ (Table 6 and Figs 2F).

Regarding P53showed that the treated group with combined SLAP+IL12+ART 3 week post infection had the highest reduction $(99.7 \%)$ (Table 7) idicating0 grade (Fig. 1e), however mild reaction in group F,G (Fig.1c,1d) in comparison with 
strong reaction in infected control group (group B) (FIG.1a).

As regard $\mathrm{Bcl} 2$;Table (8) showed that the treated group with SLAP+IL12+ART6 week post infection had the highest increase in the mean percentage of $\mathrm{Bcl}-2$ with strong reaction (Fig 3d), however group $\mathrm{H}$ tested by SLAP + IL12+ART3w post infection give negative reaction (Fig. 3e) in comparison to their corresponding infected control which give mild reaction (Fig. 3b).

Table (1) : Worm burden in different studied groups of mice

\begin{tabular}{|l|c|c|c|c|c|c|c|}
\hline Groups & B & C & D & E & F & G & H \\
\hline Mean \pm SD & $20.4 \pm$ & $14.2 \pm$ & $10.2 \pm$ & $9.5 \pm$ & $6.6 \pm$ & $4.2 \pm$ & $1.2 \pm$ \\
1.52 & 5.32 & 3.92 & 1.64 & 2.14 & 1.643 \\
\hline Reduction \% & $30 \%$ & $50 \%$ & $53.4 \%$ & $67.6 \%$ & $79.4 \%$ & $\% 94.1$ \\
\hline $\begin{array}{l}\text { Control infected versus treated } \\
\text { and/or immunized groups }\end{array}$ & $>0.05$ & $>0.05$ & $<0.05^{*}$ & $<0.01^{*}$ & $<0.01^{*}$ & $<0.001^{*}$ \\
\hline C versus D & \multicolumn{2}{|c|}{$>0.05^{*}$} & & & \\
\hline E versus F & \multicolumn{2}{|c|}{$<0.05^{*}$} & \\
\hline
\end{tabular}

Table (2) : Mean egg count/g liver in different studied groups of mice

\begin{tabular}{|l|c|c|c|c|c|c|c|}
\hline Groups & B & C & D & E & F & G & H \\
\hline Mean \pm SD & $\begin{array}{c}\mathbf{5 3 7 0 \pm} \\
\mathbf{3 0 1 . 7}\end{array}$ & $\begin{array}{c}4121.4 \pm \\
114.2\end{array}$ & $\begin{array}{c}2211.0 \pm \\
112\end{array}$ & $\begin{array}{c}1750.6 \pm \\
131\end{array}$ & $\begin{array}{c}605.3 \pm \\
42.4\end{array}$ & $\begin{array}{c}202.6 \pm \\
17.4\end{array}$ & $\begin{array}{c}15.7 \pm \\
7.2\end{array}$ \\
\hline Reduction \% & $23.3 \%$ & $58.8 \%$ & $67.4 \%$ & $88.7 \%$ & $96.2 \%$ & $99.7 \%$ \\
\hline $\begin{array}{l}\text { Control infected versus } \\
\text { immunized and/or treated groups }\end{array}$ & $>0.05$ & $<0.05^{*}$ & $<0.01^{*}$ & $<0.001^{*}$ & $<0.001^{*}$ & $<0.001^{*}$ \\
\hline C versus D & & \multicolumn{2}{|c|}{$<0.05^{*}$} & & & \\
\hline F versus E & & & & $<0.01^{*}$ & \\
\hline
\end{tabular}

Table (3) : Mean egg count /g intestine in different studied groups of mice

\begin{tabular}{|l|c|c|c|c|c|c|c|}
\hline \multicolumn{1}{|c|}{ Groups } & B & C & D & E & F & G & H \\
\hline Mean \pm SD & $\begin{array}{c}6995 \pm \\
117.2\end{array}$ & $\begin{array}{c}4996.2 \pm \\
351.2\end{array}$ & $\begin{array}{c}3107.5 \pm \\
401\end{array}$ & $\begin{array}{c}2311.7 \pm \\
342.4\end{array}$ & $\begin{array}{c}779.7 \pm \\
151.2\end{array}$ & $\begin{array}{c}352.4 \pm \\
17.4\end{array}$ & $\begin{array}{c}25.2 \pm \\
12.6\end{array}$ \\
\hline Reduction \% & $28.5 \%$ & $55.5 \%$ & $66.9 \%$ & $88.8 \%$ & $94.6 \%$ & $99.6 \%$ \\
\hline $\begin{array}{l}\text { Control infected versus } \\
\text { immunizedand/or treated groups }\end{array}$ & $<0.05 *$ & $<0.01 *$ & $<0.001 *$ & $<0.001 *$ & $<0.001^{*}$ & $<0.001 *$ \\
\hline C versus D & & \multicolumn{3}{|c|}{$<0.05 *$} & & $<0.001 *$ & \\
\hline E versus F & & & & \\
\hline
\end{tabular}


Table (4) : Mean percentage of developmental stages (mature, immature and dead) in different studied groups of mice

\begin{tabular}{|c|c|c|c|c|c|c|c|}
\hline Groups & \multirow{2}{*}{ B } & \multirow{2}{*}{$\mathbf{C}$} & \multirow{2}{*}{ D } & \multirow{2}{*}{$\mathbf{E}$} & \multirow{2}{*}{$\mathbf{F}$} & \multirow{2}{*}{ G } & \multirow{2}{*}{$\mathbf{H}$} \\
\hline Mean \pm SD & & & & & & & \\
\hline Dead ova & $10.8 \pm 2.5$ & $54.4 \pm 1.9$ & $53.5 \pm 7.5$ & $83.9 \pm 1.5$ & $93.4 \pm 1.3$ & $96.4 \pm 1.5$ & $98.4 \pm 1.7$ \\
\hline Immature ova & $27.8 \pm 10.8$ & $26.7 \pm 2.8$ & $22.5 \pm 14.6$ & $10.6 \pm 2.3$ & $3.1 \pm 2.5$ & $3.2 \pm 1.7$ & $1.2 \pm 2.3$ \\
\hline Mature ova & $61.4 \pm 12.8$ & $9.9 \pm 1.5$ & $24.0 \pm 13.2$ & $4.5 \pm 1.7$ & $2.5 \pm 1.6$ & $0.4 \pm 0.8$ & $0.4 \pm 1.2$ \\
\hline \multicolumn{2}{|c|}{$\begin{array}{l}\text { Control infected versus immunized } \\
\text { and/or treated groups(mature ova) }\end{array}$} & $<0.05^{*}$ & $<0.05^{*}$ & $<0.05^{*}$ & $<0.001 *$ & $<0.001 *$ & $<0.001 *$ \\
\hline \multicolumn{2}{|c|}{$\begin{array}{l}\text { Control infected versus immunized } \\
\text { and/or treated groups(dead ova) }\end{array}$} & $>0.05$ & $<0.05$ & $<0.01 *$ & $<0.001 *$ & $<0.001 *$ & $<0.001 *$ \\
\hline \multicolumn{2}{|c|}{$\begin{array}{l}\text { Control infected versus Control } \\
\text { infected versus immunized and/or } \\
\text { treated groups(immature ova) }\end{array}$} & $>0.05$ & $<0.05^{*}$ & $<0.05^{*}$ & $<0.001 *$ & $<0.001 *$ & $<0.001 *$ \\
\hline \multicolumn{3}{|c|}{ C versus D } & \multicolumn{2}{|c|}{$<0.01 *$} & & & \\
\hline \multicolumn{3}{|l|}{ F versus $E$} & & & \multicolumn{2}{|c|}{$<0.001 *$} & \\
\hline \multicolumn{3}{|l|}{ F versus $\mathbf{G}$} & & & & & $<0.001 *$ \\
\hline \multicolumn{3}{|l|}{ E versus G } & & & \multicolumn{3}{|c|}{$<0.001^{*}$} \\
\hline
\end{tabular}

Table (5) : Mean hepatic granuloma number in different studied groups of mice

\begin{tabular}{|l|c|c|c|c|c|c|c|}
\hline \multicolumn{1}{|c|}{ Groups } & B & C & D & E & F & G & H \\
\hline Mean \pm SD & $14.2 \pm 2.6$ & $10.3 \pm 2.4$ & $8.9 \pm 1.8$ & $5.5 \pm 1.2$ & $3.8 \pm 2.2$ & $3.2 \pm 1.4$ & $1.4 \pm 0.4$ \\
\hline Reduction \% & $13.3 \%$ & $37.3 \%$ & $61.2 \%$ & $73.2 \%$ & $77.4 \%$ & $90 \%$ \\
\hline $\begin{array}{l}\text { Control infected versus immunized } \\
\text { and/or treated groups }\end{array}$ & $>0.05$ & $<0.05^{*}$ & $<0.01^{*}$ & $<0.001^{*}$ & $<0.001^{*}$ & $<0.001^{*}$ \\
\hline C versus D & & \multicolumn{3}{|c|}{$<0.01^{*}$} & & & \\
\hline E versus F & \multicolumn{3}{|c|}{$<0.01^{*}$} & \\
\hline
\end{tabular}

Table (6) : Mean hepatic granuloma diameter in different studied groups of mice

\begin{tabular}{|l|c|c|c|c|c|c|c|}
\hline \multicolumn{1}{|c|}{ Groups } & B & C & D & E & F & G & H \\
\hline Mean \pm SD & $311.0 \pm 9.9$ & $301 \pm 12.8$ & $188.2 \pm 4.7$ & $65.8 \pm 3.5$ & $21.4 \pm 1.6$ & $11.5 \pm 0.8$ & $6.8 \pm 0.4$ \\
\hline Reduction \% & $9.9 \%$ & $39.4 \%$ & $78.8 \%$ & $93 \%$ & $96.3 \%$ & $97.8 \%$ \\
\hline $\begin{array}{l}\text { Control infected versus } \\
\text { immunized and/or treated } \\
\text { groups }\end{array}$ & $>0.05$ & $<0.05^{*}$ & $<0.01^{*}$ & $<0.001^{*}$ & $<0.001^{*}$ & $<0.001^{*}$ \\
\hline C versus D & & \multicolumn{3}{|c|}{$<0.01^{*}$} & & \\
\hline E versus F & \multicolumn{3}{|c|}{} & & $<0.01^{*}$ & \\
\hline
\end{tabular}

Table (7) : Mean percentage of P53 in different studied groups of mice

\begin{tabular}{|l|c|c|c|c|c|c|c|}
\hline \multicolumn{1}{|c|}{ Groups } & B & C & D & E & F & G & H \\
\hline Mean \pm SD & $24.2 \pm 2.5$ & $10.3 \pm 3.2$ & $6.1 \pm 2.5$ & $6.9 \pm 0.8$ & $4.2 \pm 0.8$ & $2.6 \pm 0.3$ & $0.2 \pm 0.1$ \\
\hline Reduction \% & $57.6 \%$ & $71.6 \%$ & $74.9 \%$ & $82.7 \%$ & $89.3 \%$ & $99.17 \%$ \\
\hline $\begin{array}{l}\text { Control infected versus immunized } \\
\text { and/or treated groups }\end{array}$ & $<0.01^{*}$ & $<0.01^{*}$ & $<0.001^{*}$ & $<0.001^{*}$ & $<0.001^{*}$ & $<0.001^{*}$ \\
\hline C versus D & & \multicolumn{2}{|c|}{$<0.01^{*}$} & & & \\
\hline E versus F & & & & \multicolumn{2}{|c|}{$<0.01^{*}$} & \\
\hline F versus G & & & & \multicolumn{2}{|c|}{$<0.01^{*}$} \\
\hline E versus G & & & \multicolumn{3}{|c|}{$<0.001^{*}$} \\
\hline
\end{tabular}


Table (8) : Mean percentage of Bcl2 in different studied groups of mice

\begin{tabular}{|l|c|c|c|c|c|c|c|}
\hline \multicolumn{1}{|c|}{ Groups } & B & C & D & E & F & G & H \\
\hline Mean \pm SD & $4.5 \pm 2.1$ & $6.4 \pm 1.2$ & $6.9 \pm 1.6$ & $7.4 \pm 2.4$ & $8.5 \pm 0.7$ & $9.1 \pm 0.4$ & $0.0 \pm 0.0$ \\
\hline Increase \% & $42.2 \%$ & $53.3 \%$ & $64.5 \%$ & $88.8 \%$ & $102 \%$ & $\begin{array}{c}\text { Negative } \\
\text { reaction }\end{array}$ \\
\hline $\begin{array}{l}\text { Control infected versus immunized } \\
\text { and/or treated groups }\end{array}$ & $<0.01^{*}$ & $<0.01^{*}$ & $<0.01^{*}$ & $<0.001^{*}$ & $<0.001^{*}$ & $<0.001^{*}$ \\
\hline C versus D & & \multicolumn{2}{|c|}{$<0.01^{*}$} & & & \\
\hline E versus F & & & \multicolumn{3}{|c|}{$<0.01^{*}$} & \multicolumn{2}{|c|}{$<0.01^{*}$} \\
\hline F versus G & & & \multicolumn{3}{|c|}{$<001^{*}$} \\
\hline E versus G & & & \multicolumn{3}{|c|}{} \\
\hline
\end{tabular}

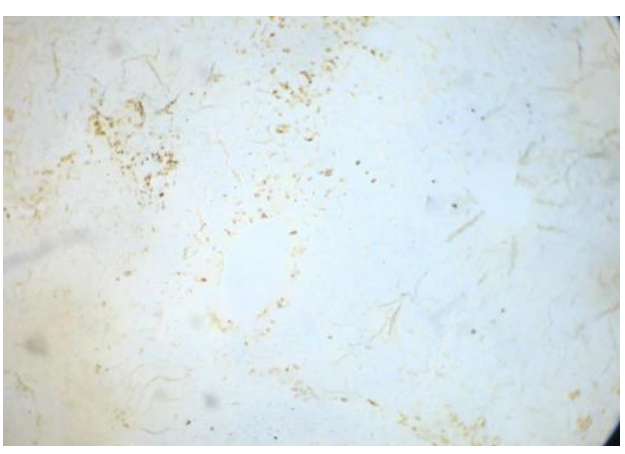

A

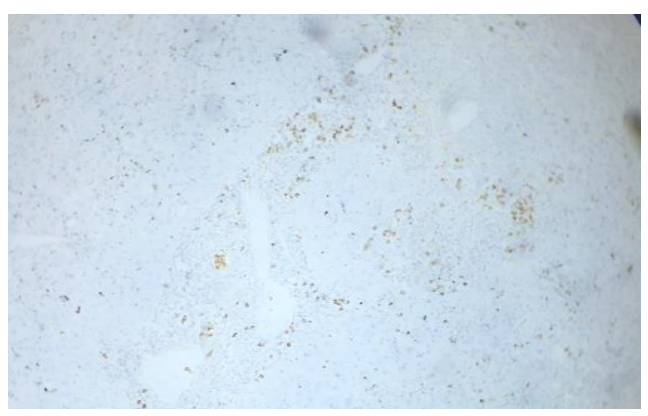

C

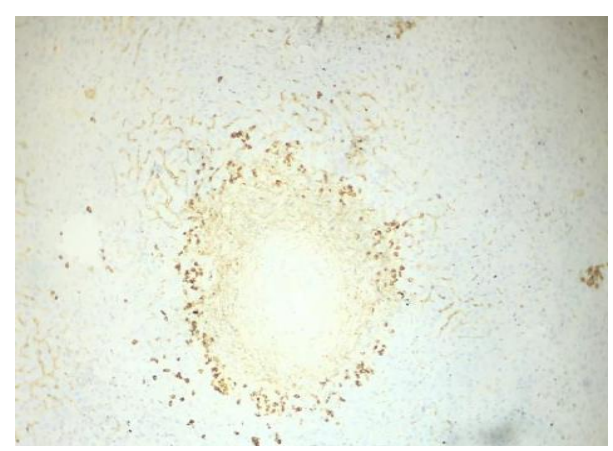

B

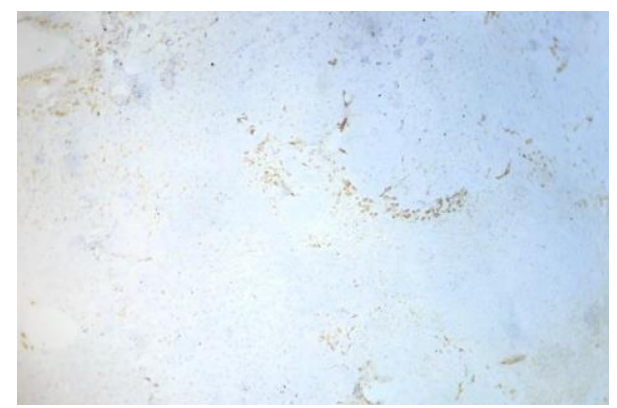

D

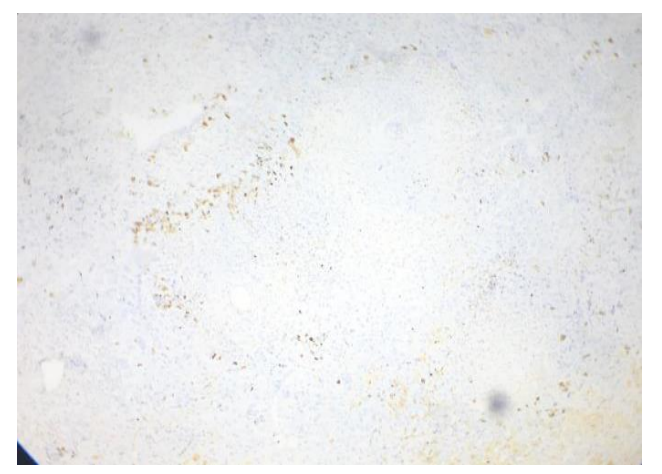

$\mathbf{E}$

Fig. (1) show the grade of reaction of P53 (X,100)(a) Cut section in the liver of group B (S. mansoni infected control group) showing strong positive reactions for P53 of grade 3, (b) represent group E showing moderate reaction for p53, (c) Represent group F showing mild reaction for P53, (d) Represent group G showing mild reaction for P53, (e) Represent group H showing 0 grade for P53. 


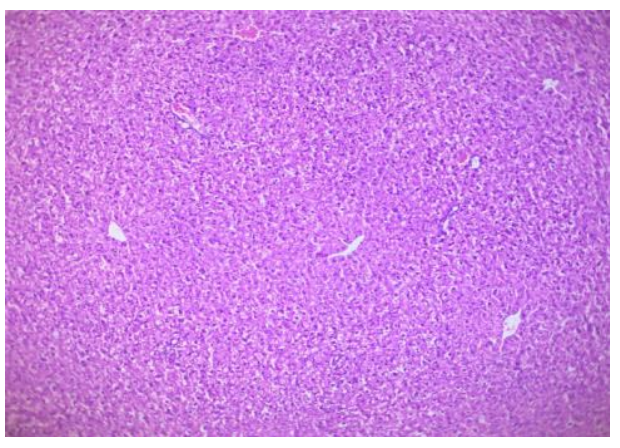

A

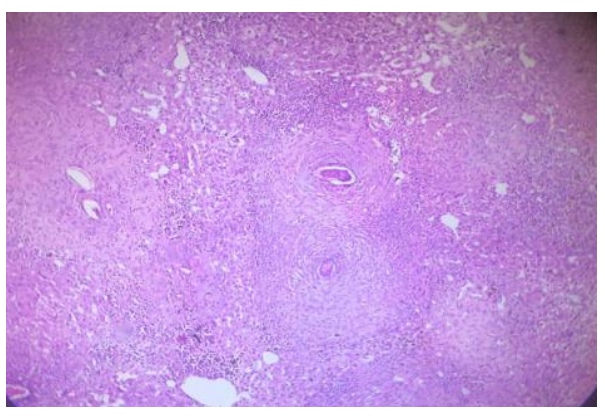

$\mathbf{C}$

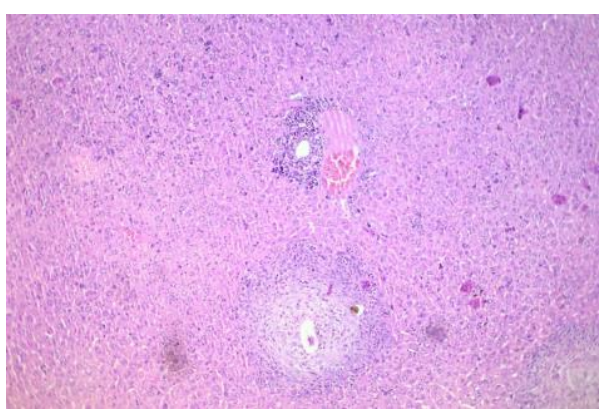

$\mathbf{E}$

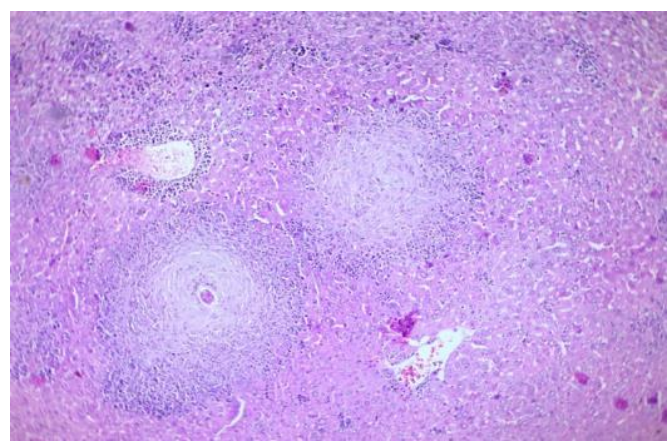

B

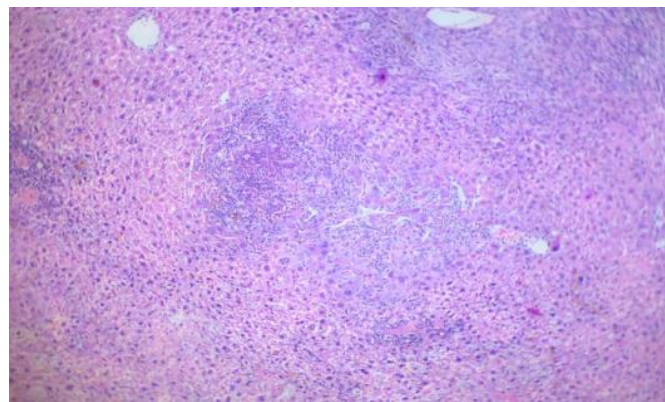

D

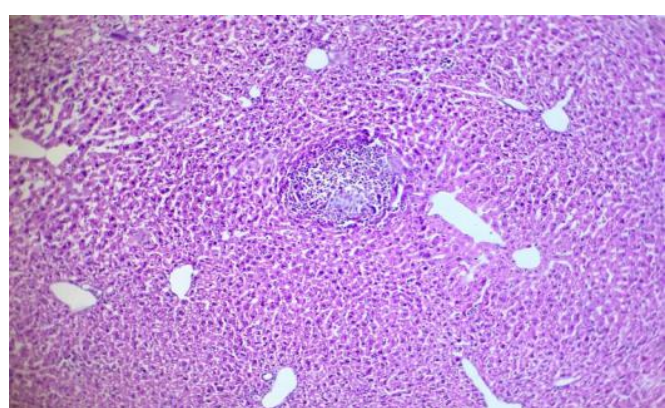

$\mathbf{F}$

Fig. (2) showing stained section in the liver with (H\&E,X100).(a) Stained section in the liver of group A (control healthy group) showing normal hepatic architecture, (b) showing group B(infected group), large multiple cellular granulomas with central fresh ova,(c) represent group E showing moderate number of granulomas with degenerated ova ,(d) Represent group F showing mild granulomas with degenerated ova, (e) Represent group $G$ showing mild granulomas with degenerated ova, (F) Represent group $\mathrm{H}$ showing few granulomas small in size with central completely degenerated ova. 


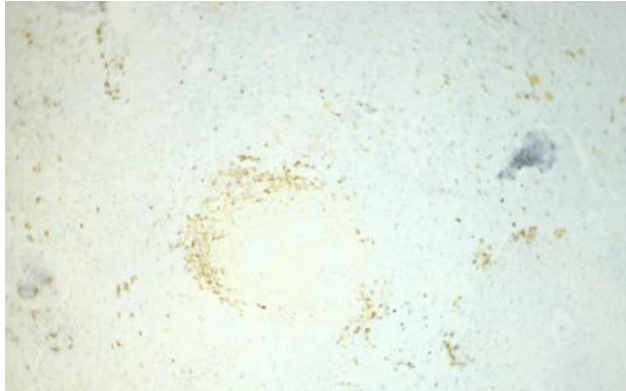

$\mathbf{A}$

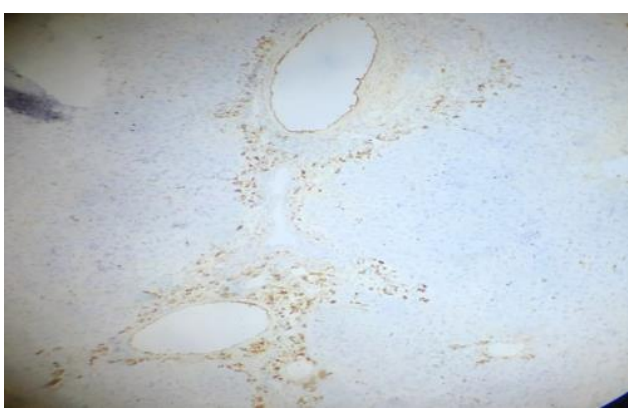

C

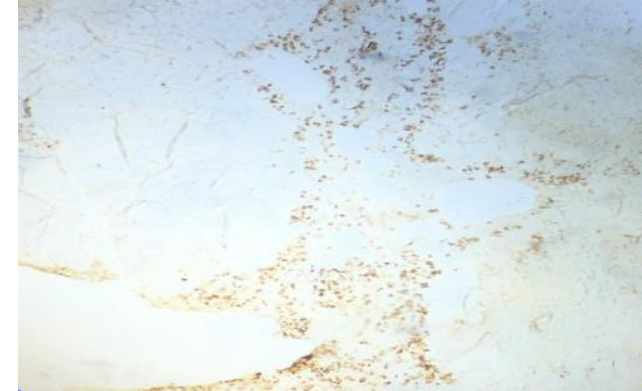

B

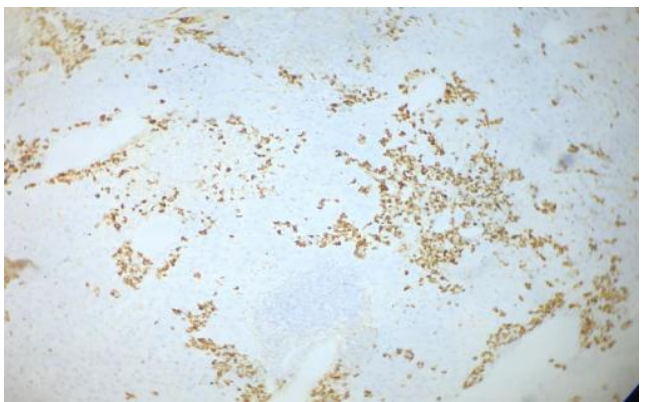

D

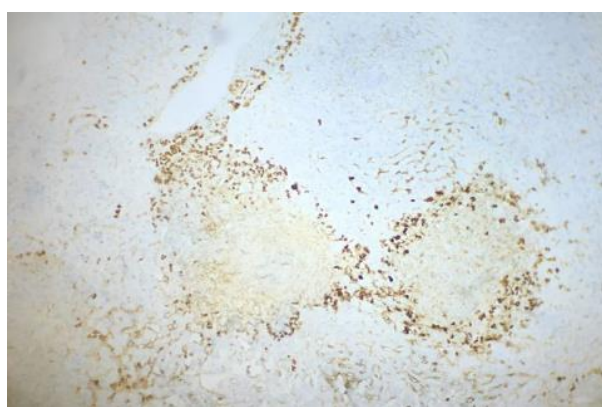

E

Fig. (3) show the grade of reaction of Bcl2 $(\mathbf{X , 1 0 0})(\mathbf{a})$ Cut section in the liver of group B (S. mansoni infected control group) showing 0 grade for Bcl2 (b) represent group E showing mild reaction for $\mathrm{Bcl} 2$, (c) Represent group $\mathrm{F}$ showing moderate reaction for Bcl2, (d) Represent group G showing strong reaction for $\mathrm{Bcl} 2$, (e) Represent group $\mathrm{H}$ showing 0 grade for $\mathrm{Bcl} 2$.

\section{DISCUSSION}

The development of resistance to the therapeutic effect of PZQ are highly increasing and this is the time for introducing a new therapeutic approach [41]. In Africa including Egypt, many studies recorded the low-cure rates of PZQ [4]. So, there is an ongoing need to develop alternative drugs to combat schistosomiasis, artemisinins are new promising antischistosomal compounds with well tolerated therapeutic dosage range [42]. Artesunate (ART) is documented to be less toxic among those several artemisinin derivatives especially when given orally due to its pharmacokinetic properties [43]. Ambitious effort have been made in the last two decades to develop an effective vaccine especially after appearance of praziquantel resistence [44]. The lung-stage schistosomulae produce high protective immunity induced by many epitopes which recall CD4+ Th1 response in vitro [45]. The present work is concerned with comparison between SLAP vaccine alone or with adjuvant (IL12) pre infection and treatment with artesunate post infection. The parameters used for evaluation were worm burden, egg count per gram of liver, egg count per gram of intestine, oogram, granuloma number and size and liver cell apoptosis (P53,BcL2). In the present study the subcutaneous routes of immunization were used because they are the best way as mentioned by Green et al. [46].

In the present work, a significant reduction in the worm burden, liver and intestine egg count, the reduction is higher in group treated with 
artesunate 3 weeks post infection than group immunized by SLAP without IL12.however the reduction increased by addition of IL12. In addition, ART monotherapy $3 \mathrm{w}$ PI resulted in a high significant reduction than ART monotherapy $6 \mathrm{w}$ PI when compared to their corresponding infected control group. A lower reduction was found in group $\mathrm{H}$ immunized by SLAP plus IL12 preinfection followed by artesunate treatment 3 weeks post infection. This indicating that artesunate have a better result at 3 weeks. These results were attributed to, the inhibitory effect of artesunate on the juvenile stages of schistosomes in early stages of infection. Since artesunate are more active against early developmental stages of schistosome [47]. This result was in agreement with Keiser et al. [48] who reported significant reductions in total worm burden of (75.6\%).On the other hand Mahmoud and Botros [49] reported anti-parasitic activity with the maximum reduction in worm burden (90\%) when ART was given at 6 weeks post-infection (PI) in addition to PZQ.

Regarding SLAP plus IL12 vaccine, it gave better results than artesunate 3 weeks post infection in the present study with reductions reaching $67.6 \%$ in worm burden, $88.7 \%$ in mean number of eggs in liver, and $88.8 \%$ in mean number of eggs in intestine. This is in agreement with Salem et al [50] who stated that IL12 is a potential adjuvant in many diseases including schistosomiasis.IL-12 increase the cell mediated immunity and Th1 cell response [17]. In another study, Feng et al. [51] reported that the worm and egg reduction rates were $64.3 \%$ and $79.9 \%$ in mice vaccinated by $S$. japonicum DNA with IL12.

In the current study, histopathological changes in the liver of infected as well as immunized groups, treated groups, or both immunized and treated, revealed that in the group immunized with SLAP plus IL12 showed significant reductions in hepatic granuloma number and diameter of $(73.2 \%$ and $93 \%)$ respectively. While there was a less significant reductions in the group treated with ART3w PI $(61.2 \%$ and 78.8) respectively compared to their corresponding control infected groups .The best reduction occurs in group $\mathrm{H}(90 \%, 97.8)$ respectively. This is in accordance with Botros and Bennett [52] who stated that the downregulation of granuloma caused by ART alone is less effective than combined with other drug.On the other hand,in another study reported that the therapeutic effect of ART even in combination therapy against schistosomiasis was inferior to that of praziquantel [53,54]. Howevermice vaccinated with SLAP plus IL-12 in the current study showed a significant anti-pathology effect by partly inhibiting granuloma formation. This is in agreement with Zhu et al. [55] who reported an increase in the protective immunity provided by $S$. japonicum Chinese strain $23 \mathrm{kDa}$. Moreover, Bu et al. [56] stated that IL-12 has a prominent role when administered as an adjuvant to DNA vaccine preparation for decreasing worm burden, granuloma number and size .

Apoptosis is an important regulator of host responses during infection with a variety of parasites [57]. Several studies suggested that antigens of $S$. mansoni can induce apoptosis in host cells [58]. Previous studies relied on immunohistochemical studies for detection of immunoreactivities of P53 [28] and Bcl-2 as apoptotic markers for evaluation of the theraputic effect of anti schistosomal drugs [3]. The present work was planned to study the hepatocyte induced apoptosis in liver tissues of experimentally infected mice with $S$. mansoniusing immunohistochemical expression of P53 and $\mathrm{Bcl}-2$ immunoreactivities.

In the current study, there was significant increase in the immunohistochemical changes and the incidence of apoptosis after S. mansoni infection, but the immunization of mice by SLAP vaccine with and without interleukin 12 or the treatment with ART 3 weeks or combination of both vaccination and treatment improved these alterations. The best result occurred in group $\mathrm{H}$ (immunized by SLAP and IL12 then treated by ART $3 \mathrm{~W}$ post infection). Also, the control healthy group in this study, was negative for both p53 and Bcl-2 which are marker of immunoreactivities. This was previously approved by Botros et al. [3] and Tosson et al. [28].

In the current work, significant reduction in the expression of P53 was observed after ART monotherapy treatment $3 \mathrm{~W}$ post infection (71.6\%) but more higher reduction in P53 was observed after immunization by SLAP+IL12 (82.7).The highest reduction in group $\mathrm{H}$ immunized by SLAP+IL12 then treated by ART $3 \mathrm{w}$ post infection (99.17). This was in accordance with Botros et al. [3] who reported that the reduction in apoptosis represented by expression of FAS protein in ART monotherapy treated group was higher than that achieved after PZQ monotherapy treatment. 
Using IL12 in the present study as an adjuvant to SLAP vaccine also proved its beneficial effect as it decreased the pathology and apoptosis of the hepatic cells. This is in agreement with Hogg et al. [59] who found that vaccination of mice with radiation-attenuated schistosomes, stimulated intense inflammatory reactions and release of pro-inflammatory IL-12, which is important for Th1-type immune responses that are partially host protective. Morever Rutizky and Stadecker [60] demonstrated that IL-23, but not IL-12, is the critical signal necessary to support the proinflammatory Th IL-17 subset involved in high pathology schistosomiasis.

On the other hand, there was a marked decrease in the mean percentage of $\mathrm{Bcl}-2$ expression in the control infected untreated group (4.5 \pm 2.1$)$ when compared to P53 expression in the same group (24.22.5). While the control healthy group showed negative (-ve) reaction for $\mathrm{Bcl}-2$. This was explained by Scorrano and Korsmeyer [61] who reported that $\mathrm{Bcl}-2$ is important in protecting the cell against the apoptosis without affecting cell proliferation, and when there is an excess of antiapoptotic proteins, the cells are more resistant to apoptosis.

In the current work, significant increase in the expression of $\mathrm{Bcl}-2$ was observed after ART monotherapy treatment $3 \mathrm{w}$ PI (53.3\%). A higher significant increase in Bcl-2 in group Fimmunized by SLAP + IL12 (88.8\%).

The highest increase in the percentage of hepatocytes expressing Bcl-2 was observed in group $\mathrm{G}$ immunized by SLAP+IL12 then treated by ART $6 \mathrm{w}$ post infection (102\%). But in group $\mathrm{H}$ immunized by SLAP+IL12 then treated by ART $3 \mathrm{w}$ post infection showed -ve reaction to Bcl-2 (similar to the reaction achieved by the control normal group), this can be explained by the prophylactic effect of SLAP +IL12 in addition to ART when taken early in schistosomiasis infection [62[. This in agreement with Botros et al. [3] who recorded that any reduction in apoptosis was accompanied by an increase in $\mathrm{Bcl}-2$ expression in all treated groups. This was explained by the fact that Bcl-2 expression (antiapoptosis) is known to possess protective properties against oxidative damage.

\section{CONCLUSION}

The results of the present study clearly demonstrate that IL12 potentiated the protective effect of $S$. mansoni SLAP vaccine. ART treated group $3 \mathrm{w}$ PI, is better than, ART treated group $6 \mathrm{w}$ PI. On comparing all tests used for assessment, the results were showing that the combined (SLAP+IL12+ ART $3 \mathrm{w}$ post infection) was the best regarding protection. Apoptosis detected by immunohistochemical studies (P53,BcL2) is a good evaluation method for the effect of schistosomicidal drugs or vaccine on affected tissues.

Funding: None.

Conflicts of interest: None.

Ethical approval:Approved .

\section{REFERENCES}

1- World Health Organization. Schistosomiasis 2016) Available at: http://www.who.int/mediacentre/ factsheets/fs115/ena. [Last accessed on 2015 Dec 31].

2- Thetiot-Laurent SA, Robert A, Boissier J, Meunier B. Schistosomiasis chemotherapy. Angew. Chem. Int. Ed. Engl. 2013;52: 7936-7956.

3- Botros SS, Hammam O, Mahmoud M, Bergquist R. Praziquantel efficacy in mice infected with PZQ non-susceptible S. mansoniisolate treated with artemether: parasitological, biochemical and immunohistochemical assessment. APMIS. 2010; 118: 692-702.

4- Doenhoff MJ, cioli D, Utzinger J. Praziquantel: mechanisms of action, resistance and new derivatives for schistosomiasis. Curr. Opin. Infect. Dis. 2008 ;21: 659-667.

5- Cioli D, Pica-Mattoccia L . Praziquantel. Parasitol. Res. 2003;90: 3-9.

6- Inyang-Etoh PC, Ejezie GC, Useh MF and InyangEtoh EC . Efficacy of artesunatein the treatment of urinary schistosomiasis, in anendemic community in Nigeria. Ann. Trop. Med. Parasitol. 2004; 98: 491-499.

7- Utzinger J, Xiao SH, Tanner M Keiser J. Artemisinins for schistosomiasis and beyond. Curr. Opin. Investig. Drugs. 2007;8: 105-116.

8- Hotez PJ, Strych U, Lustigman S, Bottazzi, ME. Human anthelminthic vaccines: Rationale and challenges. Vaccine. 2016;34,30:3549-55. 
9- Greenberg RM. New approaches for understanding mechanisms of drug resistance in schistosomes. Parasitology. 2013;20:1-13.

10- Molehin AJ, Rojo JU, Siddiqui SZ, Gray SA, Carter D, Siddiqui AA. Development of a schistosomiasis vaccine. Expert Rev Vaccines. 2016; 15,5:619-27.

11- Mountford AP, Harrop R, Wilson RA . Antigens derived from lung- stage larvae of Schistosoma mansoniare efficient stimulators of proliferation and gamma interferon secretion by lymphocytes from mice Vaccinated with attenuated larvae. Infect. Immun. 1995;63:1980-1986.

12- Mountford AP, Harrop R. Vaccination against Schistosomiasis: The case for Lung-stage Antigens. Parasitology Today. 2003;14:109-114.

13- Xu X, Zhang D, Sun W. A Schistosoma japonicum chimeric protein with a novel adjuvant induced a polarized Th1 immune response and protection against liver egg burdens.BMC Infect Dis. 2009 ;9:54.

14- Colombo MP, Trinchieri G. Interleukin-12 in antitumor immunity and immunotherapy. Cytokine Growth Factor Rev. 2002; 13:155- 68.

15- Argiro L, Henri S, Dessein H, Dessein AJ, Bourgois A. Induction of a protective immunity against Schistosoma mansoni with ovalbumincoupled Sm37-5coadsorbed with granulocytemacrophage colony stimulating factor (GM-CSF) or IL-12 on alum. Vaccine 1999; 17:13-18.

16- Gherardi MM, Ramirez JC, Esteban M . Interleukin-12 (IL-12) enhancement of the cellular immune response against human immunodeficiency virus type 1 env antigen in a DNA prime/vaccinia virus boost vaccine regimen is time and dose dependent: suppressive effects ofIL-12 boost are mediated by nitric oxide. J.Virol. 2000; 74,14:6278-86.

17- Fonseca CT, Brito CF, Alves JB, Oliveira SC. IL12 enhances protective immunity in mice engendered by immunization with recombinant 14 kDa Schistosoma mansoni fatty acid- binding protein through an IFN-gamma and TNF-alpha dependent pathway. Vaccine. 2004; 22,3-4:503-10.

18- Gan Y, Shi YE, Bu LY, Ning CX, Zhu HG. Vaccination of mice with recombinant nucleic acid vaccine encoding the integral membrane protein $\mathrm{Sj} 23$ and cytokineIL-12 elicits specific immune responses against Schistosoma japonica. Zhonghua Yi XueZa Zhi. 2005; 85: 193-98.

19- Cheng PC, Lin CN, Peng SY, Kang TF, Lee, KM. Combined IL-12 Plasmid and Recombinant SjGST Enhance the Protective and Anti-pathology Effect of SjGST DNA Vaccine Against Schistosoma japonicum. PLoS Negl Trop Dis. 2016; 10,2:30-32.
20- Fuchs Y, Steller H . Programmed cell death in animal development and disease. Cell. 2011 ;147,4: 742-758.

21- Duan Y, Gu X, Zhu D, Sun W, Chen J, Feng J. et al, Schistosoma japonicum soluble egg antigens induce apoptosis and inhibit activation of hepatic stellate cells: a possible molecular mechanism. Int. J .Parasitol. 2014;44: 217-224.

22- Jin S, Levine AJ . The P53 functional circuit. $J$. Cell Sci. 2001;114:4139-4140.

23- Ashkenazi A, Dixit VM. Death receptors: signaling and modulation. Science. 1998;281: 1305-1308.

24- Nicholson DW, Thornberry NA. Life and death decisions. Sci.2003; 299: 214-215.

25- Youle RJ, Strasser A. The BCL-2 protein family: opposing activities that mediate cell death. Nat. Rev. Mol. Cell Biol.2008; 9: 47-59.

26- Cory S, Adams JM. The Bcl2 family: regulators of the cellular life-or-death switch. Nat. Rev. Cancer.2002;2: 647-56.

27- Yuan S, Akey CW. Apoptosome structure, assembly, and procaspase activation. Structure. 2013,21: 501-515.

28- Tousson E, Beltagy DM, Abo Gazia M ,AlBehbehani B . Expressions of P53 and CD68 in mouse liver with Schistosoma mansoni infection and the protective role of silymarin. Toxicol. IndHealth,. publishedonline 16 April 2012 DOI:10.1177/0748233712442733.

29- Liang YS, John I, Bruce JI and David AB. Laboratory cultivation of schistosome vector snails and maintenance of schistosome life cycles. Proc. First. Sino. Am. Symp. 1987;1: 34.

30- Peters AP, Warren KS. A rapid method of infecting ice \& of the laboratory animalswith Schistosoma mansoni subcutaneous injection .J. Parasitol. 1969; 55:558-563.

31- Mangold BL, Knopf PM, Dean DA . The effect of assay conditions on therecovery of schistosomula from the lungs of normal and resistant rats infected with S. mansoni. J. Parasitol. 1978; 64: 813-821.

32- Call J L, Pilcher JB, Freeman JR and Tsang V C. Serum-free culturing of adult Schistosoma mansoniin dialysis bags for the production of excretory secretory antigens. J. Parasitol. 1995; 81, 5:742-746.

33- Bradford MM. A rapid and sensitive method for the quantitation of microgram quantities of protein utilizing the principle of protein-dye binding. Anal. Biochem. 1976 ;72: 248-254. 
34- Shaohong L, Kumagai T, Qinghua A. Evaluation of the anthelmintic effects of artesunate against experimental Schistosoma mansoni infection in mice using different treatment protocols. Parasitol. Int. 2006 ;55: 63-68.

35- Xiao S, Shen B, Utzinger J, Jacques C, Tanner M. Ultrastructural alterations in adult Schistosoma mansoni caused by artemether. Mem. Inst. Oswaldo. Cruz. 2002 ; 97,5: 717-724.

36- Duvall RH, DeWitt WB. Technique for recovering adult schistosomes from laboratory animals. Am. J. Trop. Med. Hyg. 1967; 16:438-486.

37- Cheever AW. Postmortem study of schistosomiasis manosniin man.Am. J. Trop. Med. Hyg. 1968;17: 38-64.

38- Pellegrino J, Oliveira C, Faria J , Cunha A . New approach tothe screening of drugsin experimental S. mansoniin mice. Am. J. Trop. Med. Hyg. 1962; 11:15-20.

39- Ali SA, Hamed MA . Effect of Ailanthus altissima and Zizyphusspina-christi on bilharzial infestation in mice: histological and histopathological studies. J. Appl .Sci. 2006; 6: 1437-1446.

40- Hsu SM, Raine L . Protein A, Avidin and biotin in immunohistochemistry. J. Histochem. Cytochem. 1981;29: 1349-1353.

41- Lee E F, Young N D, Lim N TY, Gasser R B and Fairlie W D. Apoptosis in schistosomes: toward novel targets for the treatment of schistosomiasis. Tren. Parasitol. 2014;30: 2-5.

42- Abdul-Ghani R, Loutfy N, Hassan A. Experimentally promising antischistosomal drugs: A review of some drug candidates notreaching the clinical use. Parasitol Res. 2009 ; 105: 899- 906.

43- Nontprasert A, Pukrittayakamee S, Nosten-Bertrand M, Vanijanonta S, White NJ . Studies of the neurotoxicity of oral artemisinin derivatives in mice. Am. J. Trop. Med. Hyg. 2000; 62: 409-412.

44- Kuntz AN, Davioud-Charvet E, Sayed AA et al , Thioredoxin glutathione reductase from Schistosomamansoni: An essential parasite enzyme and a key drug target. PloS Med. 2007; 4,6: 206.

45- Zhang YL, Jia K, Zhao BP. Identification of Th1 epitopes within molecules from the lung-stage schistosomulum of Schistosoma japonicum by combining prediction analysis of the transcriptome with experimental validation. Parasitol. Int. 2012; 61,4:586-93.

46- Green MI, Benacerrof B. Studies on hapten specific $\mathrm{T}$ cell immunity and suppression. Immunol Rev. 1980;50:163- 6.
47- Shuhua X, Jiqing $Y$, Jinying M, Huifang G, Peiying $\mathbf{J}$ and Tanner $M$. Effect of praziquantel together with artemether on Schistosoma japonicum parasites of different ages in rabbits. Parasitol. Int. 2000; 49: 25-30.

48- Keiser J, Vargas M, Doenhoff M J . Activity of Artemether and Mefloquine against Juvenile and Adult Schistosoma mansoniin Athymic and Immunocompetent NMRI Mice. Am. J. Trop. Med. Hyg. 2010;82,1: 112-114.

49- Mahmoud MR, Botros SS. Artemether as adjuvant therapy to praziquantel in Egyptian murine S. mansoni. J. Parasitol. 2005 ;91:175-178.

50- Salem ML, Attia WY, Al-Bolkiny YE, AlSharkawi IM, Demcheva M, Vournakis J. Using poly-N-acetyl glucosamine gel matrix to deliver IL-12 with antischistosomiasis vaccination. $J$ Infect Dev Ctries. 2010;4,5:318-28.

51- Feng Q, Hu JF, Chen H. Studies on effect enhancement of the Schistosoma japonicum DNA vaccine pVIV02-IL12-Sj23 by vegetal polysaccharides. Zhongguo Ji Sheng Chong Xue Yu Ji Sheng Chong Bing Za Zhi. 2005 ;23,6:401-3.

52- Botros S, Bennett J L . Praziquantel resistance. Exp. Opin. Drug Dis. 2007; 2: 535-540.

53- Keiser J, Utzinger J . Advances in the discovery and development of novel trematocidal drugs. Expert Opin. Drug Discov. 2007 ;2: 9-23.

54- Utzinger J, Tanner M, Keiser J. ACTs for schistosomiasis: do they act? Lancet Infect. Dis. 2010 ; 10: 579-581.

55- Zhu Y, Ren J, Da'dara A . The protective effect of a Schistosoma japonicum Chinese strain $23 \mathrm{kDa}$ plasmid DNA vaccine in pigs is enhanced with IL-12. Vaccine. 2004 ;23,1:78-83.

56- Bu LY, Shi YE, Gan Y, Zhu XH, Ning CX, Zhu, HG. Protective immunity induced by multivalent DNA vaccine of SchistosomajaponicumMr23 x 10(3) membrane antigen and IL-12 in mice ZhongguoJi Sheng Chong Xue Yu Ji Sheng Chong Bing ZaZhi. 2005;23,2:82-5.

57- Luder SK, Gross U, Lopez MF. Intracellular protozoanparasites and apoptosis: diverse strategies to modulate parasite-host interactions. Trends Parasitol. 2001; 10: 480-486.

58- Kasinathan RS, Greenberg RM. Schistosoma mansoni soluble egg antigens trigger erythrocyte cell death. Cell Physiol. Biochem. 2010 ; 26: 767 774. 
59- Hogg KG, Kumkate S, Mountford AP. IL-10 regulates early IL-12-mediated immune responses induced by theradiation-attenuated schistosome vaccine. IntImmunol. 2003; 15,12:1451-9.

60- Rutitzky LI, Stadecker MJ. CD4 T cells producing proinflammatory Interleukin-17 mediate high athology in schistosomiasis. Mem Inst Oswaldo Cruz. 2006;101(1):327-330.
61- Scorrano L, Korsmeyer SJ. Mechanisms of cytochrome c release by proapoptotic BCL-2 family members. Biochem. Biophys. Res. Commun. 2003; 304,3: 437-444.

62- Elmorshedy H, Tanner M, Bergquist NR, Sharaf S, Barakat R. Prophylactic effect of artemether on human schistosomiasis mansoni among Egyptian children: A randomized controlled trial. Acta Tropica. $2016 ; 158:$ 52-58. 